\title{
Analysis of Microarray Gene Expression Data Using Various Feature Selection and Classification Techniques
}

\author{
W. Jai Singh ${ }^{1 *}$ and R. K. Kavitha ${ }^{2}$ \\ ${ }^{182}$ Assistant Professor (SRG), Department of Computer Applications \\ Kumaraguru College of Technology, Coimbatore, India
}

\section{ABSTRACT}

The prediction and diagnosis of the cancer disease can be augmented by applying classification techniques on the microarray-based gene profiling data. It is observed that a massive data will be generated due to the regular changes that happen in the cancer producing genes among humans. Along with the vast expression of genes, only a minor fraction of it are substantially articulated. By performing analysis of microarray data, the cancer-causing genes can be identified with higher accuracy. Normally, feature selection will be performed on the data followed by the classification process. The objective of this manuscript is to select the meaningful gene features of DNA microarray data with the help of ANOVA technique thus displaying an enhanced performance of algorithms like Ada Boost, Neural Networks and Random Forest. To evaluate the suggested method, it is planned to lessen the gene count from breast and leukemia obtained from DNA microarray dataset. Investigational results on the above-mentioned datasets reveals that the gene picked by the suggested methodology displays an improved classification accuracy of Neural Networks. This work tries to draw a comparison between Neural Networks and ANOVA techniques along with classical feature selection method and more classifiers. It can be concluded that ANOVA feature selection method along with neural networks offers improved accuracy of classification. Also, the ROC reveals the excellent subclass of genes with a better classification accuracy.

KEY WORDS: ANOVA, FAST CORRELATION BASED FILTER, GENE EXPRESSION, INFORMATION GAIN, MICROARRAY DATA, NEURAL NETWORKS.

\section{INTRODUCTION}

Currently, in the field of biomedical and clinical research, the analysis of gene expression with the help of microarrays is gaining popularity. Latest developments in the field of DNA microarray provided a way to examine and assess the communication levels of several microarray data genes at the same time thus allowing generation of huge microarray data (Fan et. al. 2009). Microarrays have

\section{ARTICLE INFORMATION}

*Corresponding Author: jaisingh.w.mca@kct.ac.in Received 15th Oct 2020 Accepted after revision 7th Dec 2020 Print ISSN: 0974-6455 Online ISSN: 2321-4007 CODEN: BBRCBA

Thomson Reuters ISI Web of Science Clarivate Analytics USA and Crossref Indexed Journal

\section{Clarivate
Analytics}

NAAS Journal Score 2020 (4.31) SJIF: 2020 (7.728)

A Society of Science and Nature Publication,

Bhopal India 2020. All rights reserved.

Online Contents Available at: http//www.bbrc.in/

Doi: http://dx.doi.org/10.21786/bbrc/13.11/23 been extensively used in several aspects of biomedical investigations since this technique enabled researchers to perform enormous testing on gene patterns (Vilda et. al 2006). Availability of more dimensions in the microarray gene data makes it possible to assess every single gene in a particular environment on several categories of tissues (Zheng et.al. 2006). The property of extracting valuable data from microarray data analysis has drawn the attention of researchers from various field.

Also, this method poses the challenge of translating such data on the way to obtain a clear-cut understanding of the biological procedures and the diseases occurring in humans (Peng et.al. 2006). The highlights of this work are listed as follows: (i) Several feature selection approaches centered on statistical tests that helps to handle data of high dimensions are suggested. Information Gain, ANOVA and Correlation filter tests were used to identify suitable 
features. (ii) Once the features were chosen, several methods namely Ada Boost, Random forest and Neural Networks were used for dataset classification.

\section{MATERIAL AND METHODS}

Related Work: A novel way to diagnose cancer disease is made possible using the enormous microarray gene expression technique. With applying classification algorithms on the gene data, it is possible to identify the most useful and important genes which causes cancer disease. Treatment can be started at the appropriate time to the patients with the help of gene identification. One of the significant steps for classification is to decrease the gene data dimension. This can be achieved with the help of feature selection method. A feature selection framework that blends gene analysis with several other methods was discussed by Tan et al (Tan et.al 2008). It was decided that by blending various techniques, effective results can be obtained rather than using a single component algorithm. A MRMR minimum redundancy maximum relevance feature selection framework was developed by Peng (Ding et. al. 2005) which removed the data redundancies present in the gene data. Huang and Chow (Huang et.al. 2005) presented a meaningful gene attributes by using the information gain on a predefined class labelled data.

Mutual information was employed by Zhang et al (Zhang et. al. 2009) in the classification problems with multi labels and it was demonstrated that MIM displayed an improved classification accuracy. François et al (2007) by using the feature selection and feature transformation in his research work, showed an improvement in the strength of forward feature selection method. A greedy feature selection process employing mutual information theory was recommended by Hoque et al (2014). The approach fuses feature-feature mutual information and feature-class mutual information towards achieving an ideal sub class of features thus minimizing the redundancy and maximising the feature relevance. ShaSha et al (2014) developed unified MIM into a system which used cloud computing, to classify gene expression data. This way of data classification seemed to improve efficiency of the program with identical classification accuracy.

Proposed work: The existence of many trivial and inappropriate features in the data set lowers the analysis quality of cancer like disease. To overcome this problem, this work proposes to explore the dataset in a right perspective. A new way of microarray data classification is presented in this section. The approach consisted of a two-phase execution. Initially, the data input was preprocessed with the help of techniques like identifying missing data, normalising data, and performing feature selection. Subsequently, classification techniques were utilised for microarray data set classification into cancer or non-cancer samples. The steps followed is as given below:

(a) Collection of data: The study dataset was extracted from National Center of Biotechnology Information (NCBI GEO, http://www.ncbi. nlm.nih.gov/gds/). This data was provided as input to the proposed model for classification.

(b) Handling Missing data, data set normalization: Computation of mean value was used to fill the missing data in the dataset. Min-Max normalization was used to standardise the input feature values within the range value [0:1] (Kumar et.al. 2014).

(c) Dataset division: The dataset is split up into two types namely a training set and testing set.

(d) Feature selection: Tests namely Information Gain, ANOVA and Correlation filter were used to pick features with high-ranking significant values thereby reducing the data dimensionality.

(e) Classifier design: Ada Boost, Neural Networks and Random forest techniques were used to categorize microarray dataset.

(f) Model testing: For a chosen $\mathrm{K}$ fold value, the classifier model was tested with the help of test dataset and subsequently, classifier performance was assessed by referring to achieved accuracy.

Selection of features using ANOVA: Analysis of Variance (ANOVA) is a parametric statistical hypothesis test used for verifying whether the mean value of two or more data samples arrive from a similar distribution or not. F-test which belongs to a set of statistical tests is used to calculate the ratio among variance values obtained from two samples. The ANOVA technique is a kind of F-statistic hence called as ANOVA f-test. Analysis of Variance test is used when one of the variables under consideration is of numeric type and another one is categorical in nature. Results of the above test can be utilized for selecting features. Also, this helps in removing the target variable independent features.

Feature Selection Method with IG: Information Gain (IG) is an evaluation technique based on entropy which is commonly used in supervised learning research. IG can be used for categorical class label by using the various attribute values. IG is considered to be an essential measure for feature ranking. Provided the entropy as a criteria for impurity in training dataset $\mathrm{S}$, a measure indicating further details about $\mathrm{Y}$ supplied by $\mathrm{X}$ can be defined which is known as IG.

Feature Selection Method employing Fast Correlation Based Filter (FCBF) Two phases of FCBF is Significance analysis and Redundancy analysis.

In Significance Analysis, correlation is generally applied to examine the relevance. The relevance $r$ can be calculated using the following equation:

$$
r=\frac{\sum_{i}\left(x_{i}-\overline{x_{i}}\right)\left(y_{i}-\overline{y_{i}}\right)}{\sqrt{\sum_{i}\left(x_{i}-\overline{x_{i}}\right)^{2}} \sqrt{\sum_{i}\left(y_{i}-\overline{y_{i}}\right)^{2}}}
$$


Nevertheless, most systems in real world applications are observed to be non-linear. Correlation in a non-linear system can be determined with the help of Symmetrical Uncertainty (SU).

$$
S U=2\left[\frac{I G(X \mid Y)}{H(X) H(Y)}\right]
$$

$$
\begin{aligned}
& I G(X, Y)=H(X)-H(X \mid Y) \\
& H(X)=-\sum_{i} P\left(x_{i}\right) \log _{2} P\left(x_{i}\right)
\end{aligned}
$$

Where IG $(X, Y)$ represent the Information Gain. Following the positioning of relevant features, FCBF eliminates redundant features from selected features cantered on SU amongst feature and class, and among feature and feature. This is known as redundancy analysis.

Artificial Neural Networks (ANN) for Classification: The way in which human brain understands and manages information processing paved the way for data classification using ANN. It has the capability to cope with complicated features in the data. This characteristic helps in generalising and predicting the future cases with higher accuracy. In ANN, neurons are arranged in numerous layers. The concealed layer count and the presence of neurons in every layer is determined by the problem complexity. In order to collect predictor variables as input in the form of a node, the input layer communicates with outside environment. This information will be transferred to first hidden layer which is further multiplied by associated weights. Finally, these multiplied values are summed up and supplied through a nonlinear transfer function. The values are scaled further, and an output is produced which resembles to the axon of the neuron. Cancer classifiers based on Neural network have been employed together with binary-class and multi-class problems. This helps to identify cancer or non-cancer samples, type of cancer, or the risk of survival.

Table 1. Classification Results using Breast and Leukemia dataset with all features

\begin{tabular}{|l|c|c|c|c|}
\hline \multirow{2}{*}{ Classifier } & \multicolumn{4}{|c|}{ AUC } \\
\cline { 2 - 5 } & $\begin{array}{c}\text { Breast } \\
\text { Dataset }\end{array}$ & $\begin{array}{c}\text { Leukemia } \\
\text { Dataset }\end{array}$ & $\begin{array}{c}\text { Breast } \\
\text { Dataset }\end{array}$ & $\begin{array}{c}\text { Leukemia } \\
\text { Dataset }\end{array}$ \\
\hline Adaboost & 0.477 & 0.818 & 0.485 & 0.594 \\
\hline Neural Network & 0.710 & 0.874 & 0.670 & 0.625 \\
\hline Random Forest & 0.666 & 0.769 & 0.598 & 0.656 \\
\hline
\end{tabular}

Experimental Results and Discussions: Breast and leukemia microarray gene data are used to evaluate the various classification techniques. The Breast dataset contains a total of 97 samples with 24481 number of genes. The leukemia dataset contains 72 samples with 7219 genes. The combination of ANOVA with Neural Networks are proposed. The performance of the classifier is evaluated using classification accuracy and AUC - Area

\begin{tabular}{|c|c|c|c|c|}
\hline \multirow[t]{2}{*}{ Classifier } & \multicolumn{2}{|c|}{ AUC } & \multicolumn{2}{|c|}{ Classification Accuracy } \\
\hline & $\begin{array}{c}\text { Breast } \\
\text { Dataset }\end{array}$ & $\begin{array}{c}\text { Leukemia } \\
\text { Dataset }\end{array}$ & $\begin{array}{c}\text { Breast } \\
\text { Dataset }\end{array}$ & $\begin{array}{c}\text { Leukemia } \\
\text { Dataset }\end{array}$ \\
\hline Adaboost & 0.619 & 0.838 & 0.629 & 0.750 \\
\hline Neural Network & 0.881 & 0.973 & 0.804 & 0.844 \\
\hline Random Forest & 0.822 & 0.943 & 0.763 & 0.734 \\
\hline
\end{tabular}
Under the Curve. Table 1 to 4 shows the comparison results.

Table 3. Classification Results using Breast and Leukemia dataset with fast correlation filter

\begin{tabular}{|l|c|c|c|c|}
\hline \multirow{2}{*}{ Classifier } & \multicolumn{4}{|c|}{ AUC Classification Accuracy } \\
\cline { 2 - 5 } & $\begin{array}{c}\text { Breast } \\
\text { Dataset }\end{array}$ & $\begin{array}{c}\text { Leukemia } \\
\text { Dataset }\end{array}$ & $\begin{array}{c}\text { Breast } \\
\text { Dataset }\end{array}$ & $\begin{array}{c}\text { Leukemia } \\
\text { Dataset }\end{array}$ \\
\hline Adaboost & 0.565 & 0.704 & 0.567 & 0.562 \\
\hline Neural Network & 0.798 & 0.849 & 0.711 & 0.703 \\
\hline Random Forest & 0.866 & 0.825 & 0.773 & 0.609 \\
\hline
\end{tabular}

Table 4. Classification Results using Breast and Leukemia dataset with Information Gain

\begin{tabular}{|l|c|c|c|c|}
\hline \multirow{2}{*}{ Classifier } & \multicolumn{4}{|c|}{ AUC Classification Accuracy } \\
\cline { 2 - 5 } & $\begin{array}{c}\text { Breast } \\
\text { Dataset }\end{array}$ & $\begin{array}{c}\text { Leukemia } \\
\text { Dataset }\end{array}$ & $\begin{array}{c}\text { Breast } \\
\text { Dataset }\end{array}$ & $\begin{array}{c}\text { Leukemia } \\
\text { Dataset }\end{array}$ \\
\hline Adaboost & 0.636 & 0.804 & 0.639 & 0.703 \\
\hline Neural Network & 0.817 & 0.914 & 0.763 & 0.781 \\
\hline Random Forest & 0.853 & 0.848 & 0.753 & 0.703 \\
\hline
\end{tabular}

In Table 1, the Breast and Leukemia microarray gene expression data are categorised by Adaboost, Neural Networks and Random Forest with all gene attributes. In the Table 2, the attributes are selected by ANOVA with classification techniques is applied in the data set. In Table 3, fast correlation filter method is applied to select the features. In Table 4, Information Gain as a feature selection method. It can be seen from Tables 1 to 4, the ANOVA + Neural Networks perform better than other individual and combinational methods. Therefore, the proposed approach improves the classification performance of Breast and Leukemia microarray data. Figure 1 to 4 show the performance of the various methods and proposed method using ROC Curve. Figure 3 and 4 shows pictorial representation for the comparison 
result of Breast and Leukemia cancer dataset. The peak of the graph shows the maximum classification accuracy of these dataset by using the ANOVA + Neural Networks.

\section{CONCLUSION}

In this paper, an innovative method of microarray data classification for cancer has been suggested. The proposed method includes dimensionality reduction of gene expression data with the help of ANOVA technique, Information Gain, Neural Network and Fast Correlation filter. Research results suggest that using ANOVA with Neural Networks proves to be useful and efficient for classification.

Conflicts of Interest: The authors declare no conflicts of interest.

\section{REFERENCES}

Ding C, H. Peng (2005), Minimum redundancy feature selection from microarray gene expression data, J. Bioinf. Comput. Biol. 3 (02), 185-205.

Fan L, K.-L. Poh, P. Zhou (2009), A sequential feature extraction approach for Naïve Bayes classification of microarray data. Expert Syst. Appl. 36, 9919-9923.

François D, F. Rossi , V. Wertz , M. Verleysen (2007), Resampling methods for parameter-free and robust feature selection with mutual information, Neurocomputing 70 (7), 1276-1288 .

Hoque N, D.K. Bhattacharyya , J.K. Kalita (2014), MIFS-ND: a mutual information-based feature selection method, Exp. Syst. Appl. 41 (14), 6371-6385 .
Huang D, T.W. Chow (2005) , Effective feature selection scheme using mutual information, Neurocomputing 63, 325-343.

Kumar M, S. Kumar Rath (2014), Classification of microarray data using kernel fuzzy inference system, Int. Scholarly Res. Notices, http://dx.doi.org/ 10.1155/2014/769159.

Peng Y (2006), A novel ensemble machine learning for robust microarray data classification. Comput. Biol. Med. 36, 553-573.

Sha-Sha W, L. Hui-Juan , J. Wei , L. Chao (2014), A construction method of gene expression data based on information gain and extreme learning machine classifier on cloud platform, International Journal of Database Theory and Application 7 (2), 99-108 .

Tan F, X. Fu , Y. Zhang , A.G. Bourgeois (2008), A genetic algorithm-based method for feature subset selection, Soft Comput. 12 (2), 111-120.

Vilda P.G, F. Díaz, R. Martínez, R. Malutan, V. Rodellar, C.G. Puntonet (2006), Robust preprocessing of gene expression microarrays for independent component analysis. Independent Component Analysis and Blind Signal Separation, Springer, pp. 714-721.

Zhang M L, J.M. Peña (2009), V. Robles , Feature selection for multi-label naive Bayes classification, Inf. Sci. 179 (19), 3218-3229 .

Zheng C.H, D.-S. Huang, L. Shang (2006), Feature selection in independent component subspace for microarray data classification. Neurocomputing 69, 2407-2410. 